\title{
Cladóceros de los fiordos y canales patagónicos localizados entre el golfo de Penas y el estrecho de Magallanes
}

\author{
Paula Rosenberg \& Sergio Palma \\ Escuela de Ciencias del Mar, Pontificia Universidad Católica de Valparaíso \\ Casilla 1020, Valparaíso, Chile \\ E-mail: cladocero@yahoo.com; spalma@ucv.cl
}

\begin{abstract}
RESUMEN. Se analiza la distribución geográfica de los cladóceros Evadne nordmanni, Podon leuckarti y Pseudoevadne tergestina, capturados con redes Bongo mediante arrastres oblicuos efectuados entre $200 \mathrm{~m}$ de profundidad y superficie. Estos organismos se colectaron en 41 estaciones oceanográficas distribuidas en los fiordos y canales patagónicos ubicados entre el golfo de Penas y la boca occidental del estrecho de Magallanes. La especie más abundante fue $E$. nordmanni, seguida de $P$. leuckarti y $P$. tergestina. Esta última se encontró sólo en las estaciones ubicadas en el canal Concepción. Las mayores densidades de E. nordmanni y P. leuckarti se registraron en el canal Messier y fiordo Baker, mientras que las menores se registraron en las estaciones localizadas en aguas oceánicas. Esta distribución estuvo asociada a gradientes termohalinos, circulación estuarina y profundidad del lugar. Se determinó que los cladóceros se asociaron principalmente con la masa de Agua Subantártica Modificada.
\end{abstract}

Palabras claves: cladóceros, distribución, fiordos, canales patagónicos, Chile.

\section{Cladocerans in Patagonian fjords and channels, between the Penas Gulf and Strait of Magellan}

\begin{abstract}
In the Chilean region of Patagonian fjords and channels between the Penas Gulf and the western mouth of Magellan's strait, Evadne nordmanni, Podon leuckarti and Pseudoevadne tergestina cladocerans were identified. The analyzed organisms were captured with Bongo nets through oblique hauls between $200 \mathrm{~m}$ of depth to the surface, in 41 oceanographic stations. The most abundant species was E. nordmanni, followed by P. leuckarti and P. tergestina present only in Messier and Concepción channels. The highest densities of E. nordmanni and P. leuckarti were registered in the Messier channel and the Baker fjord, while the smallest densities corresponded to the oceanic waters stations. This distribution was associated with thermohaline gradients, estuarine circulation and depth. The association between cladocerans and the Modified Subantartic Water Mass was determinated.
\end{abstract}

Key words: cladocerans, distribution, fjords, Patagonian channels, Chile.

\section{INTRODUCCIÓN}

Los fiordos y canales australes ubicados entre el golfo de Penas y el estrecho de Magallanes constituyen un ecosistema extenso, caracterizado por condiciones oceanográficas extremas. Esta amplia zona se caracteriza por el ingreso permanente de aguas oceánicas de origen subantártico que se mezclan con aguas interiores más frías y menos salinas, provenientes de lluvias, ríos y deshielos cordilleranos de glaciares y ventisqueros (Silva et al., 1998; Guzmán $\&$ Silva, 2002). Debido a los escasos asentamientos humanos existentes en esta zona, este ecosistema de aguas interiores ha sido escasamente perturba- do; sin embargo, por sus características oceanográficas extremas, solo algunas especies planctónicas han logrado adaptarse exitosamente (Palma et al., 1999).

Las características oceanográficas de temperatura y salinidad en la zona de fiordos y canales muestran una estructura de dos capas. Una superficial (0$50 \mathrm{~m}$ ) de amplias fluctuaciones térmicas y bajos valores de salinidad, y una más profunda $(50 \mathrm{~m}$ a fondo) con un patrón térmico más estable, que tiende a una estructura cuasi homotermal (Pickard, 1971; Pinochet \& Salinas, 1996; Guerrero, 2000), donde 
la salinidad aumenta en función de la profundidad presentando también valores más estables. El grado de mezcla en los fiordos, depende entre otros factores, del caudal de las aguas aportadas por los ríos, del escurrimiento costero o derretimiento de ventisqueros, y de los vientos y mareas. Por estas razones, la temperatura y la salinidad son menores en la cabeza que en la boca de los fiordos, las cuales también dependen de la época del año y batimetría del fondo (Pinochet \& Salinas, 1996; Sievers et al., 2002).

Entre los organismos zooplanctónicos que habitan las aguas interiores se encuentran los cladóceros, que son pequeños crustáceos más frecuentes en aguas dulces que marinas. En los océanos sólo se conocen nueve especies pertenecientes a la familia Sididae, que es monoespecífica con Penilia avirostris Dana, 1852, y a la familia Podonidae, constituida por Podon leuckarti Sars, 1862, P. intermedius Lilljeborg, 1853, Pleopis polyphemoides (Leuckart, 1859), P. schmackeri (Poppe, 1889), Evadne nordmanni Lovén, 1836, E. spinifera Müller, 1867 y Pseudoevadne tergestina (Claus, 1877) (Onbé, 1999).

Debido a su pequeño tamaño y a las agregaciones estacionales que forman en áreas costeras, particularmente en primavera y verano, estos microcrustáceos son importantes en las tramas tróficas pelágicas, principalmente para pequeños consumidores secundarios del meroplancton, con quienes coexisten temporal y espacialmente (Vives, 1966; Mujica \& Espinoza, 1994; Ramírez, 1981, 1996).

Los cladóceros son cosmopolitas, comunes en aguas costeras cálidas y templadas, aunque también hay algunas especies oceánicas. En ocasiones, su distribución horizontal está relacionada con corrientes oceánicas y pueden ocupar amplias zonas biogeográficas. Por esta razón, algunas especies se consideran indicadoras de masas de agua, como $P$. avirostris que es indicadora de la influencia esporádica de la corriente Subtropical de Brasil sobre la plataforma continental del norte de Argentina (Ramírez \& De Vreese, 1974; Onbé, 1999).

Si bien no se descarta su presencia a mayor profundidad, los cladóceros son predominantemente epipelágicos. Al igual que otras especies zooplanctónicas, efectúan migraciones nictimerales, aunque en algunas especies como E. nordmanni, se ha detectado una fotoperiodicidad inversa (Ramírez \& De Vreese, 1974; Onbé, 1999). Dado que en determinados periodos del año forman quistes de resisten- cia, su ausencia en algunas áreas ha sido interpretada erróneamente (Ramírez, 1981, 1996).

En aguas chilenas, los cladóceros han sido registrados ocasionalmente (Palma \& Kaiser, 1993), señalándose solo su presencia o ausencia en muestras planctónicas (IFOP, 1984; Mujica \& Rojas, 1985; Vera et al., 1996; Avaria et al., 1999). Solamente Mujica \& Espinoza (1994), realizaron un estudio más detallado de estos organismos, donde describen la presencia y distribución de Evadne nordmanni, E. spinifera, Penilia avirostris, Pleopis polyphemoides y Pseudoevadne tergestina entre Arica y el golfo de Arauco.

En el presente trabajo se identifican las especies de cladóceros colectados en los fiordos y canales patagónicos situados entre el golfo de Penas y el estrecho de Magallanes y se analiza su distribución y abundancia con relación a las características oceanográficas de la zona.

\section{MATERIALES Y MÉTODOS}

Durante el Crucero Cimar 2 Fiordo realizado entre el 15 de octubre y el 4 de noviembre de 1996, se tomaron muestras zooplanctónicas en 41 estaciones oceanográficas distribuidas en los fiordos y canales patagónicos ubicados entre el golfo de Penas $\left(46^{\circ} 48^{\prime} \mathrm{S}\right)$ y la boca occidental del estrecho de Magallanes (514'ㅇ) (Fig. 1).

En cada estación, se obtuvieron muestras de agua con botellas Niskin a las profundidades de 0,10 , $25,50,75,100,150$ y $200 \mathrm{~m}$ para determinar la concentración de oxígeno disuelto, mediante el método de Winkler modificado por Carpenter (1965), y con un CTD Seabird 25 se registró la temperatura, salinidad y densidad del agua. El procesamiento de los datos se realizó utilizando el software SEASOFT, propio del instrumento.

Además, en cada estación se efectuaron pescas oblicuas de zooplancton con redes Bongo de 350 $\mathrm{mm}$ de abertura de malla, provistas de flujómetros OSK para estimar el volumen de agua filtrado. Las pescas se efectuaron desde un máximo de $200 \mathrm{~m}$ de profundidad a superficie y en las estaciones más someras, desde los $10 \mathrm{~m}$ del fondo. Las muestras se conservaron en una solución de agua de mar con formalina al 5\%, neutralizada con tetraborato de sodio.

Para la identificación específica se consideraron los caracteres morfológicos de los apéndices 


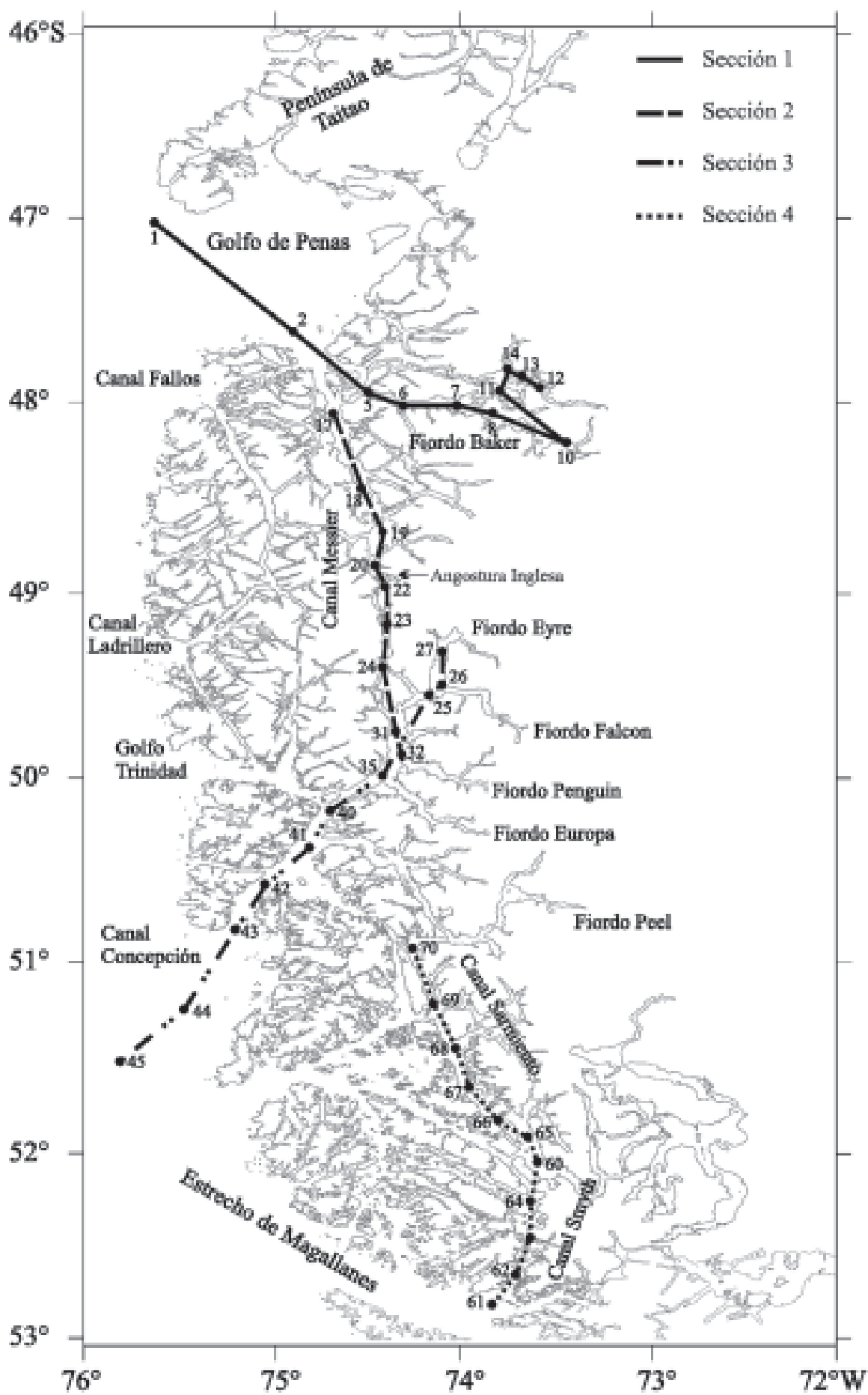

Figura 1. Ubicación de las estaciones oceanográficas en el área de estudio.

Figure 1. Locations of oceanographic sampling stations in the study area. 
torácicos, forma del caparazón y separación de los músculos elevadores de las antenas (Mujica \& Espinoza, 1994; Onbé, 1999). En cada muestra se determinó la abundancia relativa de cada especie, considerando el número normalizado de individuos por especie según la transformación $\ln (x+1)$, expresado como ind $100 \mathrm{~m}^{-3}$. Las muestras que contenían más de 200 ejemplares se subdividieron con un separador Folsom (Horwood \& Driver, 1976; Boltovskoy, 1981).

La abundancia relativa de los cladóceros en la columna de agua ( $0-200 \mathrm{~m})$ se relacionó con los promedios ponderados de temperatura, salinidad, densidad (sigma-t) y oxígeno disuelto obtenidos a profundidades discretas, utilizando el análisis de correlación canónica (Harris, 1975). La ponderación de los parámetros abióticos se efectuó en los primeros $25 \mathrm{~m}$, debido a que estos organismos se distribuyen muy superficialmente en los océanos (Villate \& Orive, 1981; Onbé \& Ikeda, 1995; Onbé, 1999).

La distribución geográfica de los cladóceros en la zona de estudio, se analizó tomando en cuenta cuatro secciones oceanográficas. En sentido esteoeste se consideró la Sección 1 (fiordo Baker al golfo de Penas) y la Sección 3 (fiordo Eyre al canal Concepción); y en sentido norte-sur la Sección 2 (golfo de Penas, canal Messier y canal Concepción) y la Sección 4 (canales Sarmiento y Smyth) (Fig. 1).

\section{RESULTADOS}

\section{Composición específica}

En el área de estudio se determinaron tres especies, Evadne nordmanni (68,6\%), Podon leuckarti $(31,3 \%)$ y Pseudoevadne tergestina $(0,1 \%)$. Las dos primeras se encontraron en la mayoría de las estaciones analizadas y constituyeron el 99,9\% de los individuos examinados, mientras que la tercera fue muy escasa y se encontró ocasionalmente.

\section{Distribución costa-océano}

En el gradiente costa-océano (Sección 1), las mayores densidades de E. nordmanni se determinaron desde el sector central hasta la boca del fiordo Baker $\left(5.774 \pm 9.414\right.$ ind $\left.\cdot 100 \mathrm{~m}^{-3}\right)$, y las menores en las cercanías de la cabeza de este fiordo y en la estación oceánica del golfo de Penas (Fig. 2). En la Sección 3, desde el fiordo Eyre y a lo largo del canal Concepción, se determinaron las menores densidades $\left(190 \pm 246\right.$ ind $\left.\cdot 100 \mathrm{~m}^{-3}\right)$, observándose un in- cremento progresivo de ejemplares hacia el océano, con un máximo cerca de la boca de este canal (estación 42).

P. leuckarti en la Sección 1 presentó algunos núcleos de mayor densidad en algunas estaciones del fiordo Baker, con máximos en la cabeza del fiordo y mínimos en el golfo de Penas. En la Sección 3, tuvo una distribución similar a E. nordmanni, caracterizada por bajas densidades a lo largo de toda esta sección $\left(143 \pm 177\right.$ ind $\left.\cdot 100 \mathrm{~m}^{-3}\right)$, particularmente en el sector oceánico (Fig. 3).

\section{Distribución norte-sur}

En la Sección 2, E. nordmanni se distribuyó formando densas agregaciones a lo largo del canal Messier $\left(9.923 \pm 12.169\right.$ ind $\left.\cdot 100 \mathrm{~m}^{-3}\right)$, registrándose un brusco descenso de abundancia en el extremo sur, en las estaciones 23 y 24 (Fig. 2). En la Sección 4 , correspondiente a los canales Sarmiento y Smyth, la abundancia fue mucho menor que en el sector norte $\left(2.714 \pm 3.247\right.$ ind $\left.\cdot 100 \mathrm{~m}^{-3}\right)$, pero igualmente se registraron algunos núcleos de alta concentración, incluso en el estrecho de Magallanes.

P. leuckarti se encontró en densidades muy inferiores a $E$. nordmanni, pero también se colectó en la mayoría de las estaciones, a excepción de aquellas ubicadas en el golfo de Penas (Fig. 3). Sus mayores densidades se registraron en algunas estaciones del fiordo Baker y en el sector central del canal Messier $\left(4.483 \pm 10.024\right.$ ind $\left.\cdot 100 \mathrm{~m}^{-3}\right)$. Al sur de los $49^{\circ} 30^{\prime} \mathrm{S}$ se detectó una fuerte disminución de abundancia, con bajas densidades en los canales Sarmiento y Smyth $\left(46 \pm 38\right.$ ind $\left.\cdot 100 \mathrm{~m}^{-3}\right)$.

P. tergestina se colectó en 5 de las 41 estaciones analizadas, encontrándose en dos estaciones del sector norte del canal Messier (estaciones 17 y 18) y en tres estaciones del canal Concepción (estaciones 40, 42 y 43), todas las cuales correspondieron a lugares con mayor aporte de aguas oceánicas subantárticas.

\section{Análisis de Correlación Canónica (ACC)}

Los resultados del ACC mostraron que en la Sección 1 (golfo de Penas-fiordo Baker), los dos componentes vectoriales fueron significativos $(\mathrm{P}<0,1)$ y tuvieron una varianza explicada de 87,12 y $12,88 \%$ para el primer y segundo vector respectivamente (Tabla 1). El primer vector explicó que el incremento de $P$. leuckarti se correlacionó con la disminución de temperatura, salinidad y densidad, e incrementos en el contenido de oxígeno disuelto. El segundo vector indicó que la disminución de E. nordmanni 


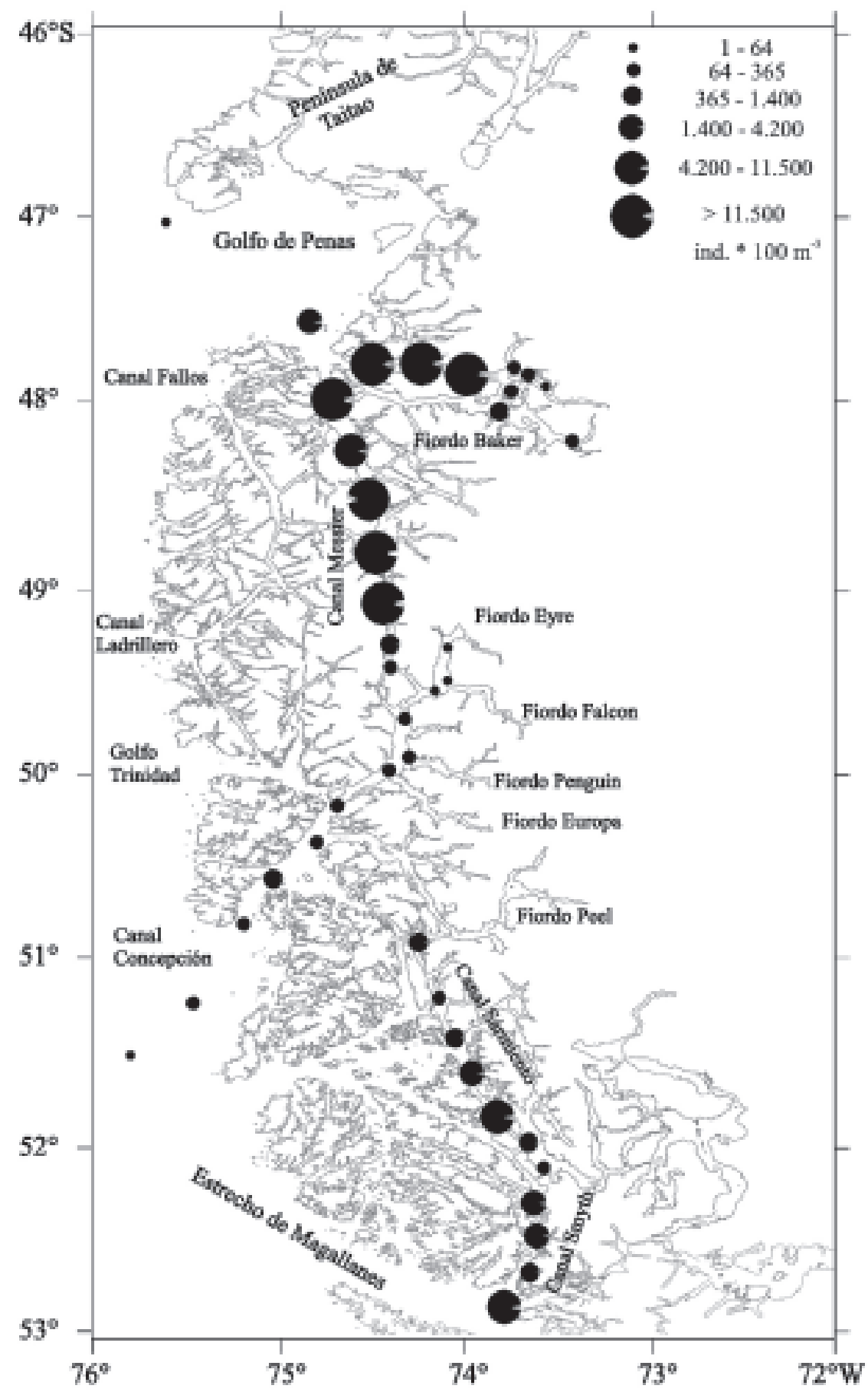

Figura 2. Distribución y abundancia de Evadne nordmanni en el área de estudio.

Figure 2. Spatial distribution and abundance of Evadne nordmanni in the study area. 


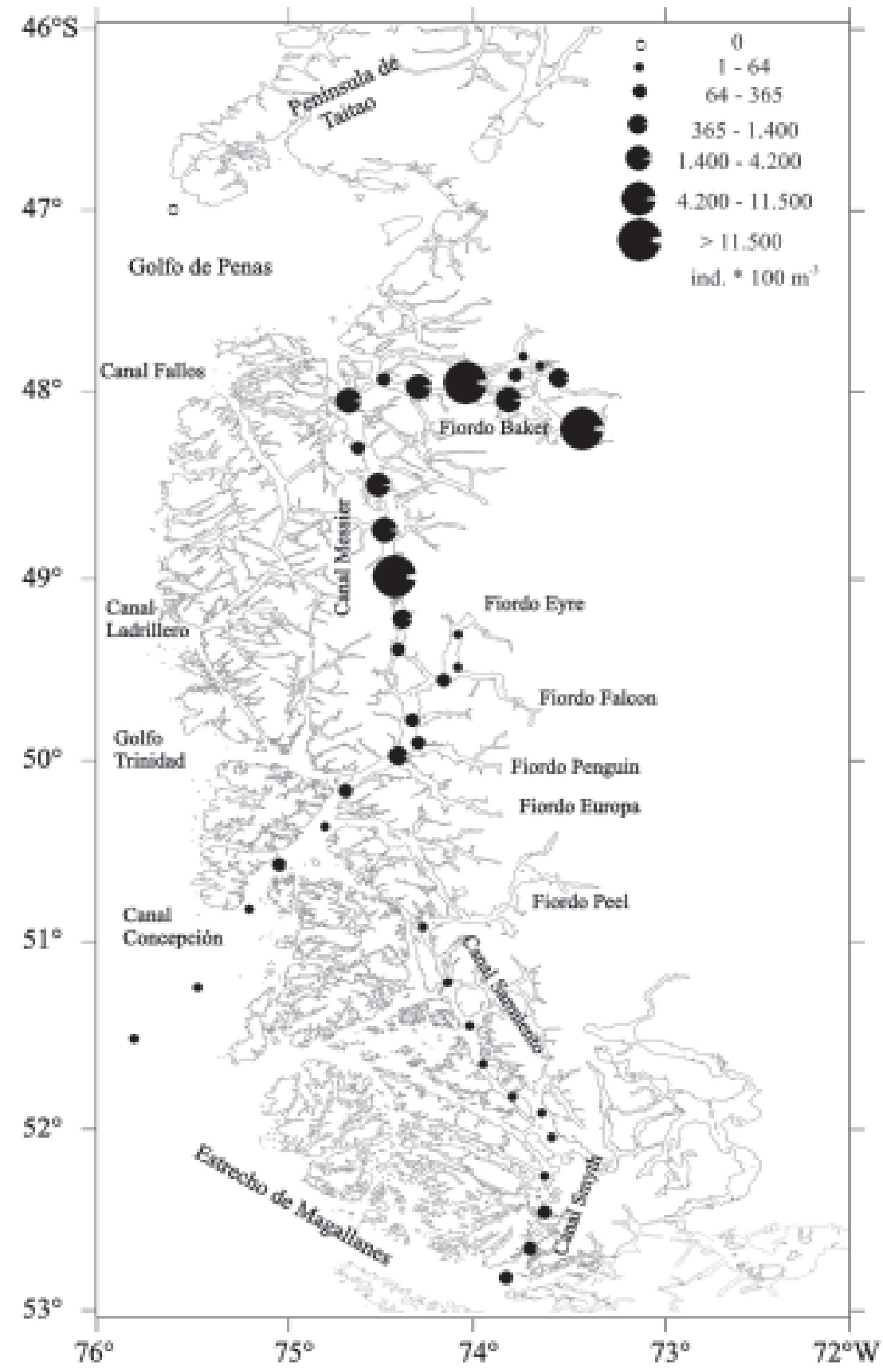

Figura 3. Distribución y abundancia de Podon leuckarti en el área de estudio.

Figure 3. Spatial distribution and abundance of Podon leuckarti in the study area. 
Tabla 1. Raíz característica, varianza explicada y valor $\mathbf{P}$ de significatividad, correspondientes a las secciones $1,2,3$ y 4 .

Table 1. Characteristic root, explained variance and P-value, corresponding to the sections 1, 2, 3 and 4.

\begin{tabular}{|c|c|c|c|}
\hline \multicolumn{4}{|c|}{ Sección 1 (golfo de Penas-fiordo Baker) } \\
\hline Vector & $\mathbf{R}$ & Varianza & $\mathbf{P}$ \\
\hline 1 & 0,916 & $87,12 \%$ & 0,012 \\
\hline 2 & 0,353 & $12,88 \%$ & 0,083 \\
\hline \multicolumn{4}{|c|}{ Sección 2 (canal Messier) } \\
\hline Vector & $\mathbf{R}$ & Varianza & $\mathbf{P}$ \\
\hline 1 & 0,994 & $55,60 \%$ & 0,001 \\
\hline 2 & 0,888 & $44,40 \%$ & 0,172 \\
\hline \multicolumn{4}{|c|}{ Sección 3 (canal Concepción-fiordo Eyre) } \\
\hline Vector & $\mathbf{R}$ & Varianza & $\mathbf{P}$ \\
\hline 1 & 0,971 & $61,63 \%$ & 0,008 \\
\hline 2 & 0,766 & $38,37 \%$ & 0,018 \\
\hline \multicolumn{4}{|c|}{ Sección 4 (canal Sarmiento-canal Smyth) } \\
\hline Vector & $\mathbf{R}$ & Varianza & $\mathbf{P}$ \\
\hline 1 & 0,827 & $94,22 \%$ & 0,004 \\
\hline 2 & 0,204 & $5,78 \%$ & 0,196 \\
\hline
\end{tabular}

se correlacionó con la disminución de temperatura y aumento en el oxígeno disuelto (Tabla 2).

En la Sección 2 (canal Messier), se obtuvieron dos componentes vectoriales, pero sólo la primera carga canónica fue significativa $(\mathrm{P}<0,1)$, con una varianza explicada del 55,60\% (Tabla 1). Este vector indicó que la disminución de $P$. leuckarti y $E$. nordmanni se correlacionó con aumentos en densidad y salinidad, y descenso en la temperatura (Tabla 2).

En la Sección 3 se obtuvieron dos vectores canónicos, ambos significativos $(\mathrm{P}<0,1)$, con una varianza explicada de $61,63 \%$ y $38,37 \%$ para el primer y segundo vector respectivamente (Tabla 1). El primer vector indicó que la disminución de $P$. leuckarti se correlacionó con aumentos en salinidad y densidad, mientras que el segundo mostró que la disminución de E. nordmanni se correlacionó con el aumento en la concentración de oxígeno disuelto y la disminución de temperatura, salinidad y densidad (Tabla 2).

En la Sección 4 (canales Sarmiento y Smyth), se obtuvieron dos componentes vectoriales, pero sólo el primero fue significativo $(\mathrm{P}<0,1)$ con una varianza explicada del 94,22\% (Tabla 1). Este análisis mostró que aumentos en salinidad y densidad,
Tabla 2. Cargas canónicas de los conjuntos de variables ambientales y biológicas correspondientes a las secciones 1, 2, 3 y 4. En negrita se indican los valores significativos.

Table 2. Canonical weights of environmental and biological variable groups corresponding to the sections 1, 2, 3 and 4. Significative values in bold.

\begin{tabular}{|c|c|c|}
\hline \multicolumn{3}{|c|}{ Sección 1 (golfo de Penas-fiordo Baker) } \\
\hline Variable & Vector 1 & Vector 2 \\
\hline Temperatura & $-0,638$ & $-0,663$ \\
\hline Salinidad & $-0,755$ & $-0,160$ \\
\hline Oxígeno & 0,788 & 0,594 \\
\hline Densidad & $-0,860$ & $-0,168$ \\
\hline E. nordmanni & 0,199 & $-0,980$ \\
\hline P. leuckarti & $\mathbf{0 , 8 8 4}$ & $-0,467$ \\
\hline \multicolumn{3}{|c|}{ Sección 2 (canal Messier) } \\
\hline Variable & Vector 1 & Vector 2 \\
\hline Temperatura & $-0,678$ & $-0,528$ \\
\hline Salinidad & 0,685 & 0,517 \\
\hline Oxígeno & $-0,070$ & 0,871 \\
\hline Densidad & 0,707 & 0,537 \\
\hline E. nordmanni & $-0,841$ & $-0,542$ \\
\hline P. leuckarti & $-0,999$ & 0,032 \\
\hline \multicolumn{3}{|c|}{ Sección 3 (canal Concepción-fiordo Eyre) } \\
\hline Variable & Vector 1 & Vector 2 \\
\hline Temperatura & $-0,326$ & $-0,871$ \\
\hline Salinidad & 0,718 & $-0,576$ \\
\hline Oxígeno & $-0,493$ & 0,611 \\
\hline Densidad & 0,755 & $-0,547$ \\
\hline E. nordmanni & $-0,431$ & $-0,902$ \\
\hline P. leuckarti & $-0,999$ & $-0,051$ \\
\hline \multicolumn{3}{|c|}{ Sección 4 (canal Sarmiento-canal Smyth) } \\
\hline Variable & Vector 1 & Vector 2 \\
\hline Temperatura & 0,294 & $-0,016$ \\
\hline Salinidad & $\mathbf{0 , 8 8 3}$ & $-0,391$ \\
\hline Oxígeno & $-0,652$ & 0,279 \\
\hline Densidad & 0,887 & $-0,400$ \\
\hline E. nordmanni & 0,998 & $-0,065$ \\
\hline P. leuckarti & $-0,711$ & 0,703 \\
\hline
\end{tabular}

unidos a un descenso en el oxígeno disuelto se correlacionaron con la disminución de E. nordmanni y P. leuckarti (Tabla 2).

\section{DISCUSIÓN}

Los cladóceros identificados, Evadne nordmanni, Podon leuckarti y Pseudoevadne tergestina, se registran por primera vez en la región de fiordos y canales patagónicos. La presencia de P. leuckarti 
constituye la primera cita de esta especie para aguas chilenas. Estos resultados permiten incrementar el conocimiento sobre la biodiversidad planctónica en la zona austral.

E. nordmanni y P. leuckarti se encontraron ampliamente distribuidas en toda el área de estudio. Estas especies son criófilas y comunes en altas latitudes del hemisferio norte, en los océanos Pacífico y Atlántico. E. nordmanni es nerítica, eurihalina (2$35 \mathrm{psu})$ y de aguas frías $\left(6-18^{\circ} \mathrm{C}\right)$, se distribuye desde el Mar del Norte hasta el Cabo de Hornos en el Atlántico sur, incluyendo el Mar Mediterráneo (Thiriot \& Vives, 1969; Thiriot, 1972-73; Ramírez, 1981; Villate \& Orive, 1981; Onbé, 1999), y en aguas chilenas se encuentra desde Arica a Talcahuano (Mujica \& Espinoza, 1994). P. leuckarti se distribuye desde Groenlandia hasta el Cabo de Hornos en el Atlántico sur (Ramírez, 1981, 1996).

En cambio, $P$. tergestina se encontró ocasionalmente en los fiordos australes y frente a la costa chilena se colectó solamente entre Arica e Iquique (Mujica \& Espinoza, 1994). Esta especie es cosmopolita y se distribuye en aguas tropicales y subtropicales entre $\operatorname{los} 40^{\circ} \mathrm{N}$ y $40^{\circ} \mathrm{S}$, siendo principalmente termófila (Ramírez, 1981, 1996; Onbé, 1999). Por lo tanto, su presencia en la región patagónica amplía su rango de distribución geográfica hasta los $51^{\circ} \mathrm{S}$ en el hemisferio sur.

La distribución de los cladóceros mostró amplias fluctuaciones de abundancia, debido a que la región de fiordos y canales patagónicos es altamente heterogénea debido a la variabilidad de sus características hidrográficas (temperatura, salinidad, densidad y oxígeno disuelto), que genera fuertes gradientes horizontales y verticales en estos parámetros (Pinochet \& Salinas, 1996; Guerrero, 2000; Sievers et al., 2002). Esta variabilidad oceanográfica afecta la distribución y abundancia de diversas poblaciones planctónicas en este sector patagónico, como se ha señalado para el fitoplancton, ictioplancton y zooplancton (Vera et al., 1996; Avaria et al., 1999; Bernal \& Balbontín, 1999; Palma et al., 1999).

Las mayores densidades de cladóceros se registraron al interior de los canales patagónicos, donde se mezclan aguas oceánicas subantárticas y aguas frescas procedentes de los fiordos. Estas altas densidades estuvieron asociadas al Agua Estuarina (131 psu) y al Agua Subantártica Modificada (32-33 psu), que predomina en la zona interior y corresponde a una mezcla lineal entre el Agua Subantártica y Agua Estuarina (Silva et al., 1998; Sievers et al., 2002). En cambio, las menores densidades se registraron en aguas oceánicas y en los canales con mayor aporte de aguas más salinas de origen subantártico. De hecho, estos organismos son comunes en aguas costeras y áreas estuarinas donde ocurren fuertes gradientes de salinidad, como desembocaduras de ríos, mares interiores y lagunas salobres (Villate \& Orive, 1981; Rodríguez \& Vives, 1984; Ramírez, 1996).

Las altas densidades registradas al norte de Angostura Inglesa en el canal Messier y en el fiordo Baker, se deberían a la circulación marina en esta área. De acuerdo a Sievers et al. (2002), desde el Baker se produce un transporte neto de agua estuarina (0-50 m) hacia el océano, que se divide en un flujo superficial que sale hacia el golfo de Penas y uno subsuperficial que ingresa al canal Messier. Bajo los $50 \mathrm{~m}$ ingresan aguas subantárticas desde el golfo de Penas hacia los canales interiores, generando una mayor estabilidad en la columna de agua y la presencia de gradientes termohalinos. Estas condiciones ambientales son favorables para el desarrollo de estos organismos (Ramírez, 1981, 1996; Villate \& Orive, 1981; Rodríguez \& Vives, 1984; Onbé, 1999)

A pesar que las especies dominantes coexisten espacialmente en la zona, éstas responden a factores ambientales diferentes. Así, los incrementos en abundancia de E. nordmanni fueron influenciados por aumentos en la salinidad y densidad del agua, y un descenso de oxígeno disuelto. En cambio, los incrementos de $P$. leuckarti se correlacionaron con la disminución de temperatura, salinidad y densidad, e incrementos en oxígeno disuelto.

La distribución espacial de cladóceros en la región patagónica presentó similitudes con los patrones de distribución de otros zooplancteres, como sifonóforos, quetognatos, eufáusidos y larvas de crustáceos decápodos; que también se encuentran formando densas agregaciones a lo largo del canal Messier y mínimos al interior de los fiordos Baker y Eyre (Palma et al., 1999; Mujica \& Medina, 2000). En este caso, la densidad de los cladóceros superó a la de larvas y macroplancton, en uno y dos órdenes de magnitud respectivamente.

Estudios efectuados en el mar Mediterráneo y océanos Pacífico y Atlántico, indican que los cladóceros presentan sus máximos estacionales en primavera-verano (Alcaraz, 1981; Villate \& Orive, 1981; Rodríguez \& Vives, 1984; Onbé \& Ikeda, 
1995; Siokou-Frangou, 1996), lo cual podría explicar las elevadas densidades registradas en este período en el sector patagónico. Probablemente, la escasa presencia de $P$. tergestina se debería a que su límite septentrional de distribución $\left(40^{\circ} \mathrm{S}\right)$, se encuentra más al norte del sector patagónico (Onbé, 1999).

\section{CONCLUSIONES}

1. Se registran por primera vez los cladóceros Evadne nordmanni, Podon leuckarti y Pseudoevadne tergestina en los fiordos y canales patagónicos chilenos. La presencia de $P$. leuckarti constituye el primer registro de esta especie para aguas chilenas.

2. Se amplía el rango de distribución geográfica de $P$. tergestina hasta los $51^{\circ} \mathrm{S}$ en el hemisferio sur.

3. E. nordmanni y P. leuckarti fueron las especies más abundantes y sus máximos ocurrieron en el canal Messier y fiordo Baker, donde estuvieron asociados a fuertes gradientes termohalinos, característicos del Agua Estuarina y Agua Subantártica Modificada.

4. La abundancia E. nordmanni se correlacionó positivamente con la salinidad y densidad del agua, y negativamente con la concentración de oxígeno disuelto. En cambio, la abundancia de $P$. leuckarti se correlacionó positivamente con el oxígeno disuelto y negativamente con la temperatura, salinidad y densidad.

\section{AGRADECIMIENTOS}

Los autores agradecen al Comité Oceanográfico Nacional y a la tripulación del buque oceanográfico AGOR "Vidal Gormaz" que permitieron la realización del Crucero Cimar 2 Fiordo, al aporte del Proyecto FONDEF 2-41 y al Prof. Nelson Silva de la Escuela de Ciencias del Mar, quien facilitó la información oceanográfica. Además, agradecen las valiosas sugerencias de los evaluadores del trabajo.

\section{REFERENCIAS}

Alcaraz, M. 1981. Ciclo anual de los cladóceros y ostrácodos planctónicos en la plataforma continental de Vizcaya (Punta Endata). Inv. Pesq., 45(1): 316.
Avaria, S., L. Jorquera, P. Muñoz \& P. Vera. 1999. Distribución de microfitoplancton marino en la zona de aguas interiores comprendida entre el golfo de Penas y el estrecho de Magallanes, Chile, en la primavera de 1996 (Crucero Cimar-Fiordo 2). Cienc. Tecnol. Mar, 22: 81-110.

Bernal, R. \& F. Balbontín. 1999. Ictioplancton de los fiordos entre el golfo de Penas y estrecho de Magallanes y factores ambientales asociados. Cienc. Tecnol. Mar, 22: 143-154.

Boltovskoy, D. (ed.). 1981. Atlas del zooplancton del Atlántico sudoccidental y métodos de trabajo con el zooplancton marino. Instituto Nacional de Investigación y Desarrollo Pesquero (INIDEP), Mar del Plata, 933 pp.

Carpenter, J.H. 1965. The Chesapeake Bay Institute technique for the Winkler dissolved oxygen method. Limnol. Oceanogr., 10: 141-143.

Guerrero, Y. 2000. Distribución de temperatura, salinidad y oxígeno disuelto en las aguas interiores de la zona de los canales australes, entre el golfo de Penas y seno Almirantazgo. Tesis de Oceanografía, Pontificia Universidad Católica de Valparaíso, $96 \mathrm{pp}$.

Guzmán, D. \& N. Silva. 2002. Caracterización física y química y masas de agua en los canales australes de Chile entre boca del Guafo y golfo Elefantes (Crucero Cimar Fiordo 4). Cienc. Tecnol. Mar, 25(2): 45-76.

Harris, R. 1975. A primer of multivariate statistic. Academic Press, New York, 332 pp.

Horwood, J \& R. Driver. 1976. A note on theorical subsampling distribution of macroplankton. J. Cons. Int. Explor. Mar., 36(3): 274-276.

Instituto de Fomento Pesquero (IFOP). 1984. Estudio del zooplancton del norte de Chile durante febrero y marzo de 1983. Rev. Com. Perm. Pacífico Sur, 15: 263-267.

Mujica, A. \& O. Rojas. 1985. Composición y abundancia del zooplancton del norte de Chile. Crucero MARCHILE XV-ERFEN VI, diciembre 1983. Cienc. Tecnol. Mar, 9: 31-52.

Mujica, A. \& E. Espinoza. 1994. Cladóceros marinos chilenos (18 $\left.30^{\circ}-37^{\circ} 30^{\prime} S\right)$. Rev. Chil. Hist. Nat., 67: 265-272.

Mujica, A. \& M. Medina. 2000. Distribución y abun- 
dancia de larvas de crustáceos decápodos en el zooplancton de los canales australes. Proyecto Cimar-Fiordo 2. Cienc. Tecnol. Mar, 23: 49-68.

Onbé, T. 1999. Ctenopoda y Onychopoda (= Cladocera). En: D. Boltovskoy (ed.). South Atlantic Zooplankton. Backhuys Publishers, Leiden, 1: 797813.

Onbé, T. \& T. Ikeda. 1995. Marine cladocerans in Toyama Bay, southern Japan Sea: seasonal occurrence and day-night vertical distributions. J. Plankton Res., 17(3): 595-609.

Palma, S. \& K. Kaiser. 1993. Plancton marino de aguas chilenas. Ediciones Universitarias de Valparaíso, Valparaíso, $151 \mathrm{pp}$.

Palma, S. \& S. Rosales. 1997. Sifonóforos epipelágicos colectados entre Puerto Montt y la-

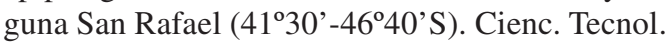
Mar, 20: 1-20.

Palma, S., R. Ulloa \& L. Linacre. 1999. Sifonóforos, quetognatos y eufáusidos de los canales australes entre el golfo de Penas y el estrecho de Magallanes. Cienc. Tecnol. Mar, 22: 111-142.

Pickard, G.L. 1971. Some physical oceanographic features of inlet of Chile. J. Fish. Res. Bd. Can., 28: 1077-1106.

Pinochet, P. \& S. Salinas. 1996. Estructura térmica y salina de fiordos y canales adyacentes a Campos de Hielo Sur, Chile. Cienc. Tecnol. Mar, 19: 93122.

Ramírez, F. 1981. Cladocera. En: D. Boltovskoy (ed.). Atlas del zooplancton del Atlántico sudoccidental y métodos de trabajo con el zooplancton marino. Instituto Nacional de Investigación y Desarrollo Pesquero (INIDEP), Mar del Plata, pp. 533-542.

Ramírez, F. 1996. Cladocera. En: R. Gasca \& E. Suárez (eds.). Introducción al estudio del zooplancton marino. El Colegio de la Frontera Sur (ECOSUR)/CONACYT, México, pp. 191-211.

Recibido: 25 agosto 2002; Aceptado 24 enero 2003
Ramírez, F. \& P. De Vreese. 1974. Taxonomía y distribución de los cladóceros en un sector de la plataforma bonaerense y adyacencias. Physis, Buenos Aires, 33(87): 511-526.

Rodríguez, V. \& F. Vives. 1984. Ciclo anual de los cladóceros en el puerto de Málaga. Inv. Pesq., 48(2): 223-233.

Sievers, H., C. Calvete \& N. Silva. 2002. Distribución de características físicas, masas de agua y circulación general para algunos canales australes entre el golfo de Penas y el estrecho de Magallanes (Crucero Cimar-Fiordo 2), Chile. Cienc. Tecnol. Mar, 25(2): 17-43.

Silva, N., C. Calvete \& H. Sievers. 1998. Masas de agua y circulación general para algunos canales australes entre Puerto Montt y Laguna San Rafael, Chile (Crucero Cimar Fiordo 1). Cienc. Tecnol. Mar, 21: 17-48.

Siokou-Frangou, I. 1996. Zooplankton annual cycle in a Mediterranean coastal area. J. Plankton Res., 18(2): 203-223.

Thiriot, A. 1972-73. Les cladocères de Méditerranée occidentale. III. Cycle et répartition a Banyuls-surMer (Golfe du Lion). Synthèse des années 1965 1969. Vie Milieu, 23(2B): 243-295.

Thiriot, A. \& F. Vives. 1969. Evadne nordmanni Lovén en Méditerranée occidentale. Vie Milieu, 20(1B): 145-157.

Vera, P., S. Avaria \& P. Muñoz. 1996. Composición y distribución del fitoplancton de los fiordos adyacentes a Campos de Hielo Sur y su relación con algunos parámetros oceanográficos. Cienc. Tecnol. Mar, 19: 73-92.

Villate, F. \& E. Orive. 1981. Abundancia y distribución de las poblaciones de cladóceros en el estuario de Plencia. Inv. Pesq., 45(2): 327-336.

Vives, F. 1966. Zooplancton nerítico de las aguas de Castellón (Mediterráneo occidental). Inv. Pesq., 30: 49-166. 